\title{
An Online Application for Retinoblastoma Surveillance
}

\author{
Nakul Singh Meghan J. DeBenedictis Arun D. Singh \\ Cleveland Clinic Cole Eye Institute, Cleveland, $\mathrm{OH}, \mathrm{USA}$
}

\section{Keywords}

Retinoblastoma - Genetics · Surveillance · Familial relationship risk of germline $R B 1$ mutation determines ophthalmic surveillance recommendations. The tool may have most value in regions where access to specialized care is limited.

(c) 2020 S. Karger AG, Base

\section{Introduction}

Retinoblastoma (RB) occurs when both copies of $R B 1$, a tumor suppressor gene, in a somatic cell of the developing retina undergo mutations. The first mutation may be inherited (either by a familial germline mutation or a de novo germline mutation) and the second mutation occurs spontaneously, or both the first and second mutations may occur spontaneously [1]. Thus, the three genetic subtypes can be referred to as familial RB, nonfamilial heritable $\mathrm{RB}$, and sporadic $\mathrm{RB}$, respectively (Table 1). Each genetic subtype has specific implications for the risk of RB in family members.

The first step in recommending appropriate surveillance is to estimate the risk of harboring a germline $R B 1$ mutation. The sensitivity of genetic testing may vary based on phenotype, whether or not a tumor sample is available, and the laboratory performing the analysis [2]. When a genetic test is not available (genotype status), the clinician must use clinical features (phenotype) of RB to stratify the risk. Specifically, all bilateral probands have a

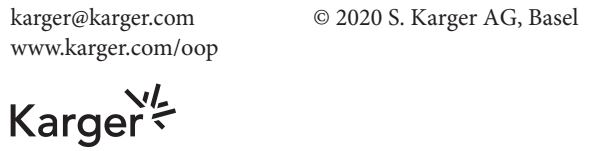

Arun D. Singh, MD

Department of Ophthalmic Oncology, Cleveland Clinic Cole Eye Institute 9500 Euclid Avenue, Desk i32 Cleveland, OH 44195 (USA)

singha@ccf.org 
Table 1. Genetic subtypes of retinoblastoma: distribution and phenotypic correlation

\begin{tabular}{|c|c|c|c|c|c|c|}
\hline & Family history & Heritability & First mutation & Second mutation & Phenotype* & Distribution \\
\hline Familial & present & heritable & inherited germline & somatic & bilateral or unilateral & $10 \%$ \\
\hline Nonfamilial & absent & heritable & new-onset germline & somatic & bilateral or unilateral & $25 \%$ \\
\hline
\end{tabular}

* Germline mutation can manifest as no tumor (carrier status), unilateral, or bilateral tumors. While the majority of unilateral cases $(85 \%)$ are sporadic in origin, $15 \%$ of unilateral tumors can be due to underlying germline mutation.

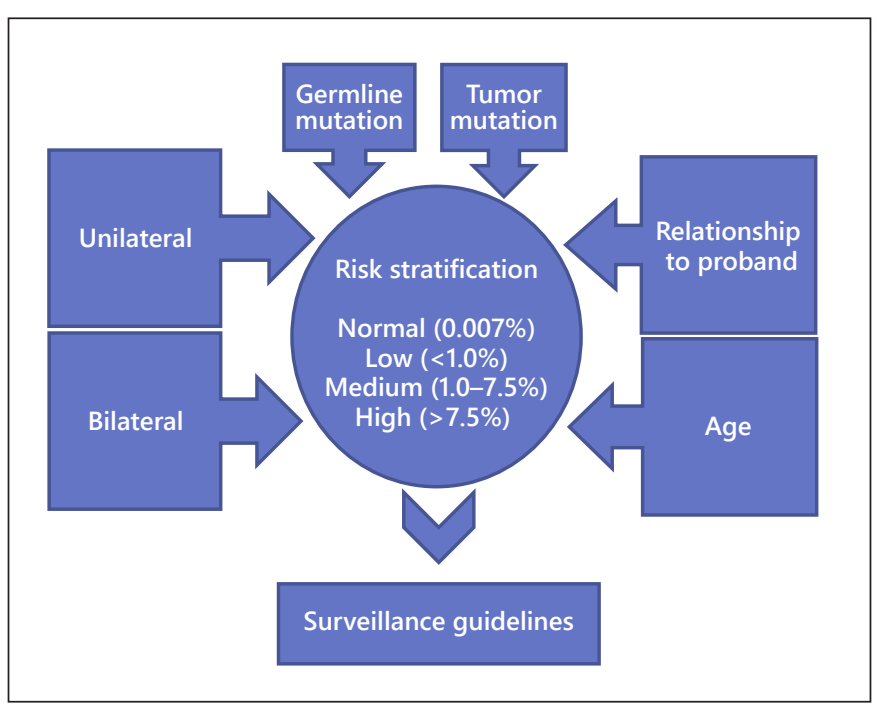

Fig. 1. Input variables (germline mutation, tumor mutation, relationship to proband, and age) for generating surveillance recommendations used in this online application.

germline mutation. Unilateral cases may either have a germline mutation that has not manifested as bilateral disease ( $15 \%$ of cases) or the RB was caused by spontaneous mutations ( $85 \%$ of cases) [3-5].

Therefore, whether a genetic test is available or not, the proband's risk of having a germline $R B 1$ mutation can be simply calculated with application of Mendelian genetics (autosomal dominant). While inheriting a germline RB1 mutation confers a high risk of developing RB (penetrance is approximately $90 \%$ ), about $10 \%$ of such cases will not develop RB (carrier status) [6-8].

Based on clinical features (phenotype), $R B 1$ mutation status (genotype), and relationship data, the consensus panel of the American Association of Ophthalmic Oncologists and Pathologists stratified the risk profile (normal population risk [0.007\%], low risk $[<1.0 \%]$, intermediate risk [1.0$7.5 \%]$, and high risk [ $>7.5 \%]$ ) of developing RB [9]. The recent surveillance guidelines proposed varying frequency and intensity of examinations (clinical vs. examination under anesthesia). While experienced clinicians or ocular oncologists can manage RB families based upon their own clinical experience [10], much of the world wherein RB occurs does not have access to such expertise [11].

To assist clinicians, we created an online application that integrates age, phenotype, genotype, and relationship data with best practice guidelines to provide specific surveillance recommendations for individuals and families with $\mathrm{RB}$.

\section{Methods}

The risk of harboring a germline $R B 1$ mutation can be estimated by the status of genetic test results (mutation detected, undetected, not tested). In the case where a genetic test was done, we allowed for the possibility that the test may fail to detect a germline mutation either due to technical limitation or germline mosaicism (false-negative rate of 3\%; Impact Genetics, Toronto, ON, Canada) $[12,13]$. In the case where a genetic test was not done, we obtained estimates for rates of germline $R B 1$ mutation in unilateral RB (15\%) from Draper et al. [6]. The rates of unaffected carriers (10\%) was obtained from Musarella and Gallie [3]. The risk of family members of having germline $R B 1$ mutation was stratified to the strata as used in the consensus report: general population risk $(0.007 \%)$, low risk $(<1.0 \%)$, intermediate risk (1.0-7.5\%), and high risk (>7.5\%) [9]. Surveillance guidelines were abstracted from the consensus report of the American Association of Ophthalmic Oncologists and Pathologists [9].

A table based on possible input variables (germline mutation, tumor mutation, relationship to proband, and age) and surveillance recommendations was constructed as a model for the online application (Fig. 1). The online application was programmed using the shiny framework [14].

\section{Results}

The online application is available at https://nakul-singh. shinyapps.io/RB_Screening_rec/ (Fig. 2). The surveillance recommendations depend on the $R B 1$ germline mutation status (detected, undetected, not tested), which are further 
Fig. 2. Surveillance recommendation without genetic test results $(\mathbf{a}, \mathbf{b})$ and with genetic test results $(\mathbf{c}, \mathbf{d})$. The risk stratification ranges from high (a), intermediate (b), low (c) to general population risk (d). The surveillance recommendation for an 11month-old child of a bilateral proband is examinations under anesthesia every month (a), for a 65-month-old sibling of a bilateral proband nonsedated it is eye examination every 6 months (b), for a 38-month-old first cousin of a proband for whom the germline and tumor mutation has been identified it is to perform nonsedated eye examination every 6 months until genetic test in the cousin can be undertaken (c), and for a 38-month-old first cousin of a proband for whom the tumor mutation, but not the germline mutation, has been identified it is nonsedated eye examination every 6 months. Genetic test in the cousin is not recommended (d). RB, retinoblastoma.

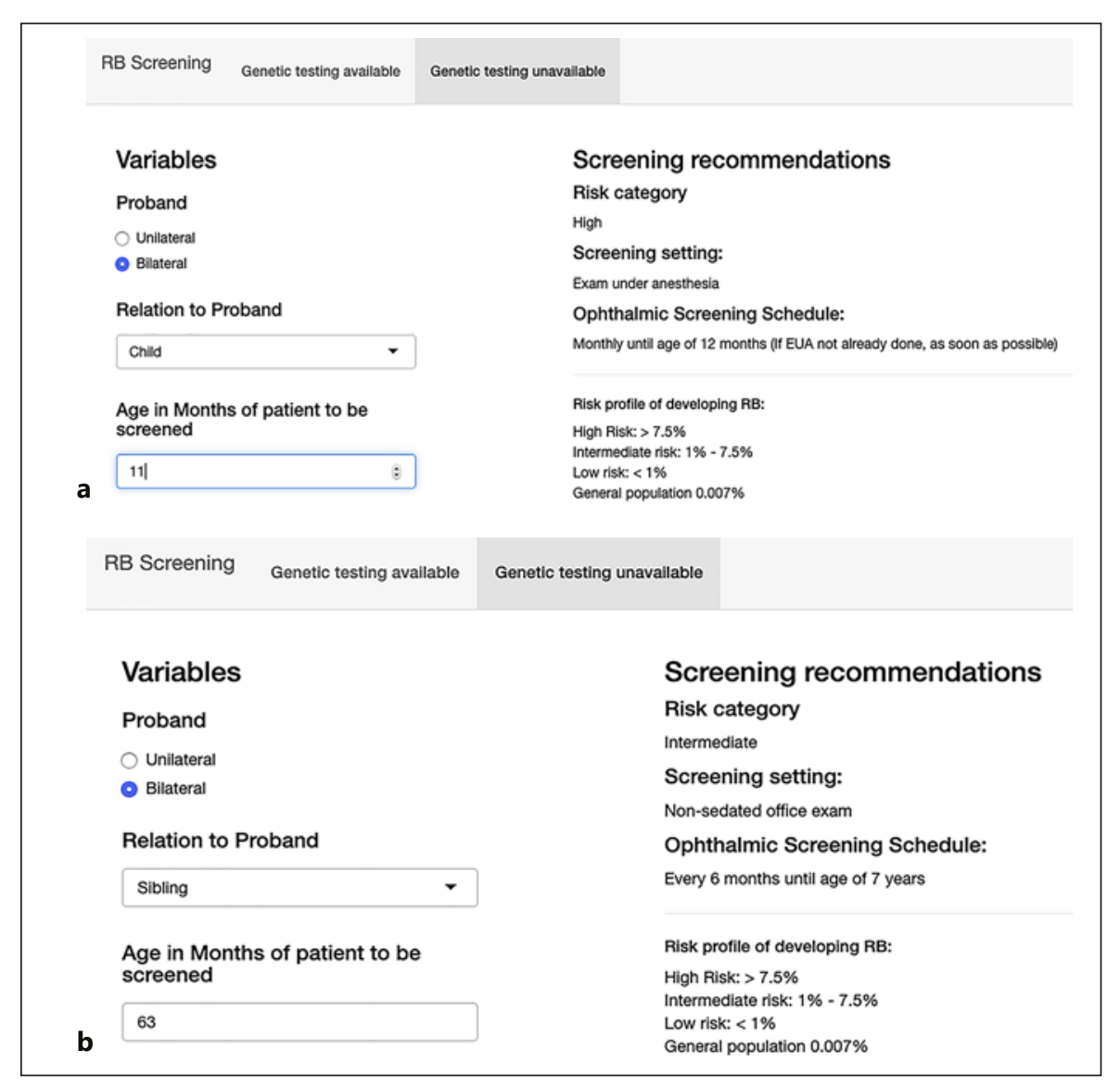

(Figure continued on next page.) subcategorized by phenotype, relationship to proband, and family structure. The surveillance recommendations for high, intermediate, low, and general population risk range from screening every 2-4 weeks to general population screening guidelines. After the age of 7 , screening for retinoma is recommended. The frequency of examination under anesthesia includes the time to first examination under anesthesia, which should be performed as soon as possible.

\section{Discussion}

$\mathrm{RB}$ is a diagnosis that may affect the entire family including parents, siblings, aunts, uncles, cousins, and future offspring. Based on the genetic test status and phenotypic aspects of the proband (first diagnosed case in the family), it may be that either the entire family or no one else in the family requires periodic ophthalmic surveillance. The ultimate purpose is early detection of RB, which offers higher globe salvage rates [15], good visual outcomes [16], and remains a top research priority [17].
We present a free online application that integrates phenotypic, genetic, and familial relationships with clinical best practice guidelines for surveillance of individuals at risk of developing $\mathrm{RB}$. Recommendations that take into account genetic test information more decidedly stratify family members into high or general population risk groups. When a genetic test result is not known and the proband is unilateral, the surveillance recommendations necessarily reflect the uncertainty of the germline mutation status. This online application in no way constitutes medical advice and is to be used as a general guide.

In cases where $R B 1$ germline status is confirmed or suspected, the risk of having germline mutation decreases proportional to the degree of relation to the proband, e.g., a child of the proband has a much higher risk than a first cousin. Surveillance intensity ranges from examinations by a general pediatrician to examinations every 2-4 week in the eye clinic or monthly under anesthesia. Undue burden is placed on families and healthcare systems to perform examinations under anesthesia when it is not indicated. 




It has been established that integrating genetic tests for germline $R B 1$ mutation in the care plan makes delivery of care cost efficient not only in developed nations such as the USA [18] and Canada [19], but also in developing nations such as India [20]. Our online application may serve as a means to efficiently allocate resources even when a genetic test is not available. The tool may have most value in regions where access to specialized ocular oncology care is limited.

\section{Statement of Ethics}

Our report complies with guidelines for human studies and was conducted ethically in accordance with the World Medical Association Declaration of Helsinki. As the study did not involve any human subjects or existing databases, ethics approval from institutional review board was not required.

An Online Application for Retinoblastoma Surveillance

\section{Disclosure Statement}

The authors declare no relevant conflicts of interest.

\section{Funding Sources}

The Cole Eye Institute is supported by an unrestricted grant from Research to Prevent Blindness. The sponsor or funding organization had no role in the design or conduct of this research.

\section{Author Contributions}

All named authors meet the International Committee of Medical Journal Editors criteria for authorship for this article, take responsibility for the integrity of the work as a whole, and gave their approval for this version to be published. 


\section{References}

1 Knudson AG Jr. Mutation and cancer: statistical study of retinoblastoma. Proc Natl Acad Sci USA. 1971 Apr;68(4):820-3.

2 DeBenedictis MJ, Singh AD. Retinoblastoma: genetic counseling and testing. In: Berry JL, Kim JW, Damato BE, Singh AD, editors. Clinical ophthalmic oncology, 3rd edition, chapter 9. New York: Springer Nature; 2019. p. 107-17.

3 Musarella MA, Gallie BL. A simplified scheme for genetic counseling in retinoblastoma. J Pediatr Ophthalmol Strabismus. 1987 MayJun;24(3):124-5.

4 Dudgeon J. Unilateral retinoblastoma - genetic implications. Br J Ophthalmol. 1996 Mar;80(3):193.

5 Notis CM, Niksarli K, Abramson DH, DeLillo AR, Ellsworth RM. Parents with unilateral retinoblastoma: their affected children. $\mathrm{Br} \mathrm{J}$ Ophthalmol. 1996 Mar;80(3):197-9.

6 Draper GJ, Sanders BM, Brownbill PA, Hawkins MM. Patterns of risk of hereditary retinoblastoma and applications to genetic counselling. Br J Cancer. 1992 Jul;66(1):2119.

7 Matsunaga E. On estimating penetrance of the retinoblastoma gene. Hum Genet. 1980; 56(1):127-8.
8 Gallie BL. Gene carrier detection in retinoblastoma. Ophthalmology. 1980 Jun;87(6): 591-5.

9 Skalet AH, Gombos DS, Gallie BL, Kim JW, Shields CL, Marr BP, et al. Screening Children at Risk for Retinoblastoma: Consensus Report from the American Association of Ophthalmic Oncologists and Pathologists. Ophthalmology. 2018 Mar;125(3):453-8.

10 Abramson DH. Re: Skalet et al.: Screening children at risk for retinoblastoma: consensus report from the American Association of Ophthalmic Oncologists and Pathologists (Ophthalmology. 2018;125:453-458). Ophthalmology. 2018 Sep;125(9):e63-4.

11 Kivelä T. The epidemiological challenge of the most frequent eye cancer: retinoblastoma, an issue of birth and death. Br J Ophthalmol. 2009 Sep;93(9):1129-31.

12 Soliman SE, Racher H, Zhang C, MacDonald H, Gallie BL. Genetics and Molecular Diagnostics in Retinoblastoma - an Update. Asia Pac J Ophthalmol (Phila). 2017 Mar-Apr; 6(2):197-207.

13 Smith JH, Murray TG, Fulton L, O’Brien JM. Siblings of retinoblastoma patients: are we underestimating their risk? Am J Ophthalmol. 2000 Mar;129(3):396-8.

14 Chang W, Cheng J, Allaire JJ, Xie Y, McPherson J, RStudio, et al. shiny: web application framework for R. 2017. https://CRAN. $\mathrm{R}$-project.org/package $=$ shiny .
15 Al-Nawaiseh I, Ghanem AQ, Yousef YA. Familial Retinoblastoma: Raised Awareness Improves Early Diagnosis and Outcome. J Ophthalmol. 2017;2017:5053961.

16 Imhof SM, Moll AC, Schouten-van Meeteren AY. Stage of presentation and visual outcome of patients screened for familial retinoblastoma: nationwide registration in the Netherlands. Br J Ophthalmol. 2006 Jul;90(7):875-8.

17 https://rbcanadaresearch.wordpress.com/ top10priorities/.

18 Dhar SU, Chintagumpala M, Noll C, ChévezBarrios P, Paysse EA, Plon SE. Outcomes of integrating genetics in management of patients with retinoblastoma. Arch Ophthalmol. 2011 Nov;129(11):1428-34.

19 Richter S, Vandezande K, Chen N, Zhang K, Sutherland J, Anderson J, et al. Sensitive and efficient detection of RB1 gene mutations enhances care for families with retinoblastoma. Am J Hum Genet. 2003 Feb;72(2):253-69.

20 Joseph B, Shanmugam MP, Srinivasan MK, Kumaramanickavel G. Retinoblastoma: genetic testing versus conventional clinical screening in India. Mol Diagn. 2004;8(4): 237-43. 